\title{
Control System Research and Performance Prospect of Low Impact
}

\section{Docking System}

\author{
Lingxuan Zhang $^{1, a *}$, Jingming Shao ${ }^{1, b}$, Huaiwu Zou ${ }^{1, c}$
}

\author{
${ }^{1}$ Aerospace System Engineering Shanghai. No.3805, Jindu Road, Minhang District, Shanghai \\ 201108, China; \\ a amyindut@hotmail.com, ${ }^{\mathrm{b}} 40337764 @ q q . c o m,{ }^{\mathrm{c}}$ shanshuijun@163.com
}

\begin{abstract}
Keywords: LIDS; System composition; Control strategy; Force compliance control
Abstract: Low Impact Docking System is an active control docking system with functions of docking and berthing, which represents the development direction of the next generation of space docking mechanism. In this paper, the system composition and operating principle of LIDS were introduced and the basic functions of its control system at each stage of the docking mission were analyzed. Moreover, the core control strategy of the control system was presented. In addition, the prospect of other operating modes and performance which LIDS could adapt to was also studied.
\end{abstract}

\section{Introduction}

Space rendezvous and docking technology is very important for the space technology development and human space exploration enhancement, it provides a huge possibility for long-term manned space flight, in-orbit construction and maintenance for the mega space facility, lunar and deep space manned-exploration and other large-scale space missions ${ }^{[1]}$.

Space docking system is a mechanism to combine two space crafts in orbit and to connect them into a whole structurally. Through the implementation of 921 program China has mastered the space rendezvous and docking technology. The Androgynous Peripheral Assembly System (APAS) has been successfully applied to the docking of the Shenzhou spacecraft and Tiangong No. $1^{[2-4]}$, which helped China to become the third country after the United States and Russia to completely-own and independently-master the space rendezvous and docking technology. However, our existing APAS technology and solutions are still insufficient to meet the demand for diversified types of space missions and scalability of exploration in the future, such as: complexity of mechanical system, longer development cycle; being lack of flexibility of buffering capacity, limited adaptation of objectives and tasks; bigger docking impact force, and etc. Therefore, in order to meet the demand for the reliability and flexibility of the space exploration in the future, space docking system has to follow the path into better adaptation and flexibility.

In order to improve the flexibility of the docking system and to simplify the mechanical system, NASA and ESA developed Low Impact Docking System (LIDS) based on the experience of APAS design and deployment ${ }^{[5]}$. This design baseline is the mechanical-electronic integration, leveraging the Active Compliance Collision Force Control Technology, it made the complete active compliant controlling docking possible, and this technology provides better adaptations to space crafts and docking capability, as well as less docking impact force during the process. Soft percussive docking technology represents the next generation of space docking and the new trend of mechanical-electronic integration in space technology. Hence, design and development of soft percussive docking technology is very important for enhancing the adaptation of docking tasks and meet the future demand of China manned space exploration.

Low impact docking system is the highly integrated mechanical-electronic controlling system, whose docking performance relies upon controlling system performance and wholesome controlling strategy. In this article, firstly, the system configuration and theory of soft percussive docking 
technology would be covered in accordance with its application scenarios and functions; secondly, the core control technology centered around soft percussive docking technology; at last, research and vision of other application scenarios and working models of soft percussive docking technology.

\section{System composition of LIDS}

To fulfill the docking tasks, LIDS mainly relies on its two subsystems: soft docking system and hard docking system. The soft docking system is to achieve the flexible capture of two vehicles and buffer the residual energy using force compliance control strategy, which is the core and key technology of LIDS. The hard docking system is to achieve the missions of hauling close the mating frame, rigid connection and locking, pressing and sealing up. The above working principles are as same as those in APAS, which are directly referred, so that they would not be discussed in this paper.

The design idea of the soft docking system of LIDS is to use a highly integrated mechatronics system to simulate a spring-damping system with adjustable cushioning performance. In docking process, the gesture of the docking ring can be real-time adjusted based on the docking impact force. Therefore, the soft docking system has to include a force sensor, a motion controller, actuator elements, and capture elements which are used to achieve the soft connection of the docking mechanisms. Based on the above ideas, the structure of the soft docking system uses the configuration of Stewart parallel mechanism. The upper and lower platform of the parallel mechanism are respectively composed by the docking ring with guide plates and the mating frame. The six kinematic branch chains are composed by six linear electro-mechanical actuators (LEMA), which are driven and controlled independently. In the internal of the docking ring, six axis force sensor is embedded in to obtain the collision force information. Because the docking collision impact is low, the electromagnetic lock is used to achieve the flexible connection after soft capture. The basic structure and system composition are shown in Fig.1 (Fig.1 presents the diagram of LIDS developed by ESA, as known as IBDM).

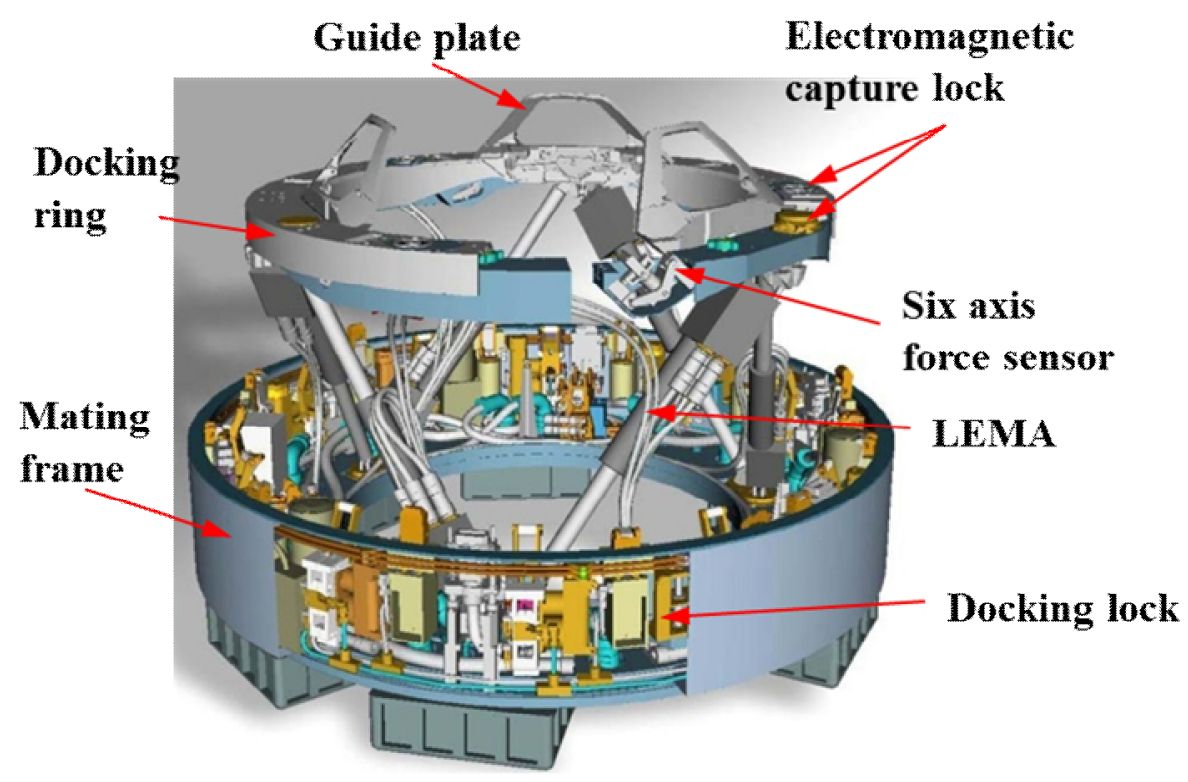

Fig. 1 Basic structure and system composition of LIDS

The system composition shows that LIDS has greatly reduced the complexity of the mechanical system of the docking system. However, this will inevitably put forward very high request to its control system. Hence, the difficulties and key technology of the docking mission achievement is 
the effective force compliance control of the docking mechanism.

\section{Operating principle of control system}

\section{Function requirement}

The outstanding feature of LIDS is using effective control to replace complex mechanism. Therefore, the performance of the control system and correlative control strategy are the key technology of LIDS to achieve docking tasks. The main functions of LIDS control system at each stage of docking process are as followed:

1) Docking preparation: The control system receives docking preparation command and then push the docking ring up to the corresponding position to meet and hold the ring under the docking initial conditions.

2) Capture and damping: In conditions of the initial approaching velocity between two vehicles and the residual energy after soft connection, the active and passive docking rings contact each other and the force sensor embedded in the active docking ring obtains the impact information. This information is the input of the control system. Through force compliance algorithm, the control system gives the gesture adjustment strategy of the active docking ring. Then, the six LEMAs execute the corresponding action to achieve the ring gesture adjustment thus complete the collision force compliance and the residual energy damping.

3) Hauling close: After the damping process, the control system receives the hauling close command. The six LEMAs retract to haul the active docking ring back in order to make active and passive docking frames mating. Because the hauling back velocity is very slow, the hauling force can be ignored.

According to the above task analysis, the control structure diagram can be gained as Fig.2, in which, the main difficulty is the effective force compliance control at capture and damping stage. For force compliance control, the involved core strategies are docking ring trajectory planning, inverse kinematics, compliance control algorithm and close-loop control of LEMA.

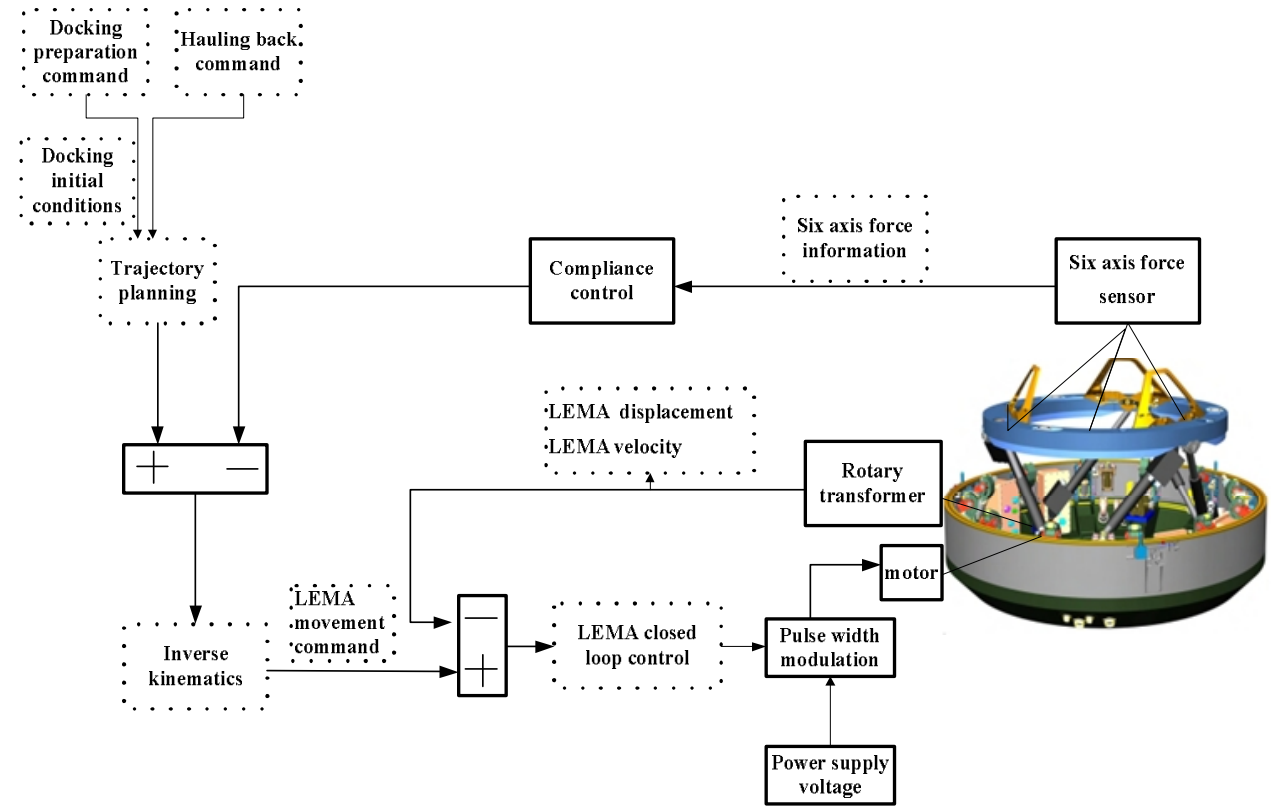

Fig. 2 The control structure diagram of LIDS

\section{Main control strategy}

1) Trajectory planning strategy

Low impact docking system is a Steward platform with 6 DOF, the track control can be reached simultaneously in all the 6 DOFs, which would meet the boundary conditions when initial time $\mathrm{t}=0$ 
and initial condition met time $\mathrm{t}=\mathrm{T}_{\mathrm{k}}$. In order to ensure the continuity and smoothness of velocity and accelerated velocity, the 3-order polynomial and 5-order polynomial trajectory planning algorithm could be employed to simultaneously plan the tracks in all the 6 DOFs in the Cartesian Space for the low impact docking system ${ }^{[6-7]}$.

The docking ring tracking planning strategy establishment of the low impact docking system should take the initial condition realization into consideration as well as system intervention and pillar force. Hence, in the planning, mathematical model based on dodging constrains should be developed according to mechanical subsystem design of the low impact docking system. It is also required to perform the multi-purpose optimization on docking ring tracks based on pull/push force, power consumption and time.

2) Inverse kinematics

Docking ring inverse kinematics is to mapping the desired docking ring position onto the 6 pillars, and then to adjust the docking ring into target position by 6 LEMA movements indirectly. Because the low impact docking system employs Stewart parallel mechanism, the docking ring position reverse engineering can be reached by applying parallel mechanism kinematical reverse algorithm.

3 ) Force compliance control

Low impact docking system could degrade system stiffness by compliant controlling strategy in order to adapt docking captive requirement. This strategy could be based upon resistance controlling method, which provides a good spring resistance features, and provides adaptation to different stiffness designs during the phases of docking capturing and buffer adjusting.

\section{Low impact docking system next steps}

1) Compliance Controlling Strategy based on Docking Ring Position

Except for the previous mentioned solution, the adjustment of the low impact docking system compliance controlling strategy is required when the 6 dimension sensor allocation design is employed (in which scenario, the force sensors are located in the 6 LEMAs, the axis direction force on the pillar could be measured directly). This type of strategy can be designed via a combination of forward and backward solutions of docking system kinetics.

2) Dual-Positive Docking

Dual-positive docking is available thanks to the low impact docking system features as controlling smart system with flexibility and kinetic controllability. This docking solution could provide backups for APAS system, better reliability of docking tasks, as well as buffer capability improvement of the docking system for heavier space craft docking mission.

3 ) Docking and Positive Capture Mode

Low impact docking system can not only simulate spring resistance system to capture and buffer the docking passively, but also perform assistant docking leveraging mechanical arms or rotating devices as docking port, thanks to the adjustable system buffer performance which allows it to minimize the system stiffness while docking. Besides, when the 0 clash is required, low impact docking system can perform precise positive capture as parallel mechanical arms/hands in the pending mode, thanks to the visual positioning technology.

\section{Conclusions}

Low impact docking system is the next generation docking system, representing the future trend of space system. It employs mechanical-electronic controlling buffering system to perform compliance adaptation and energy buffering between active and passive systems by force compliance 
controlling technology. This article researched and discussed about the core controlling strategy of such system based on the explanation of system configuration and theory, this article also analyzed the future docking mode and controlling strategy concerning the features of the low impact docking system. The research content and methodology of this article would benefit the low impact docking system future research as well as the research of related mechanical-electronic controlling compliance mechanical system.

\section{References}

[1] Jianping Zhou. Space rendezvous and docking technology[M]. National Defense Industry Press. 2013. In chinese

[2] Renzhang Zhu, Zheng anbo, Lou Hanwen, Wang Xiaoguang. A Review of The Development of Spacecraft Coupling System[J]. Manned Spaceflight. 2007(1):13-23. In Chinese

[3] Baodong Chen, Ping Tang. The Technology Development of Docking Mechanism System[J]. Aerospace Shanghai.2005(5):6-8. In Chinese

[4] Chen Baodong, Zheng Yunqing, Shao Jiming, Chen Meng. Development Of Docking Subsystem[J]. Aerospace Shanghai.2011,28(6):1-6. In Chinese

[5] B. Paijmans, K. De Vriendt, H. Dittmer,el. The International Berthing Docking Mechanism ... A New European Docking System[C].63th International Astronautical Congress, 2012,B3,7,9,X15451,Naples, Italy.

[6] J J.Craig. Introduction To Robotics Mechanics And Control[M]. China Mechine Press.2006. In Chinese

[7] Zixing Ca. Robotics[M]. Tsinghua University Press. 2000. In Chinese 